\title{
Visceral obesity and cell cycle pathways serve as links in the association between bisphenol $A$ exposure and breast cancer
}

\author{
TSU-NAI WANG ${ }^{1,2^{*}}$, PEI-JING YANG ${ }^{1}$, YU-TING TSENG ${ }^{1}$, YI-SHAN TSAI $^{1}$, PO-LIN KUO $^{2,3}$, \\ CHIEN-CHIH CHIU ${ }^{2,4}$, SHIH-SHIN LIANG ${ }^{4,5}$, TSUNG-HUA HSIEH ${ }^{6}$, \\ MING-FENG HOU ${ }^{3,7-10}$ and EING-MEI TSAI ${ }^{2,6,11,12^{*}}$ \\ ${ }^{1}$ Department of Public Health, College of Health Science; ${ }^{2}$ Research Center for Environmental Medicine; \\ ${ }^{3}$ Graduate Institute of Clinical Medicine, College of Medicine; ${ }^{4}$ Department of Biotechnology, College of Life Science, \\ Kaohsiung Medical University, Kaohsiung 80708; ${ }^{5}$ Institute of Biomedical Science, College of Science, \\ National Sun Yat-Sen University, Kaohsiung 80424; Departments of ${ }^{6}$ Obstetrics and Gynecology, and \\ ${ }^{7}$ Surgery; ${ }^{8}$ Cancer Center, Kaohsiung Medical University Hospital, Kaohsiung 80756; ${ }^{9}$ Department of Surgery, \\ Kaohsiung Municipal Siaogang Hospital, Kaohsiung 81267; ${ }^{10}$ Department of Biological Science and Technology, \\ National Chiao Tung University, Hsin-Chu 30010; ${ }^{11}$ Center for Research Resources and Development; \\ ${ }^{12}$ Graduate Institute of Medicine, College of Medicine, Kaohsiung Medical University, Kaohsiung 80708, Taiwan, R.O.C.
}

Received January 23, 2018; Accepted August 16, 2018

DOI: $10.3892 / \mathrm{ol} .2020 .11553$

\begin{abstract}
It has been identified that bisphenol A (BPA) exposure causes developmental toxicity in breast cells. However, the exact molecular mechanisms underlying the association between exposure to BPA and breast cancer remain unclear. The aim of the present study was to investigate the BPA-regulated signaling pathways associated with the aggressiveness and the development of breast cancer. Microarray technology and functional gene set analyses were used to evaluate BPA and breast cancer-associated biomarkers and pathways in a discovery-driven manner. Using individual dataset analyses, it was indicated that two BPA-associated gene sets, the visceral obesity pathway, involved in visceral fat deposits and the metabolic syndrome, and the cell cycle pathway, involved in cyclins and cell cycle regulation, were significantly associated with a
\end{abstract}

Correspondence to: Dr Tsu-Nai Wang, Department of Public Health, College of Health Science, Kaohsiung Medical University, 100 Shin-Chuan 1st Road, Kaohsiung 80708, Taiwan, R.O.C.

E-mail: wangtn@kmu.edu.tw

Dr Eing-Mei Tsai, Research Center for Environmental Medicine, Kaohsiung Medical University, 100 Shin-Chuan 1st Road, Kaohsiung 80708, Taiwan, R.O.C.

E-mail: tsaieing@yahoo.com

*Contributed equally

Abbreviations: BPA, bisphenol A; GEO, Gene Expression Omnibus; IPA, Ingenuity Pathway Analysis; MetSyn, the metabolic syndrome; ER, estrogen receptor

Key words: pathway, bisphenol A, breast cancer, gene sets, microarray high grade of aggressiveness and the development of estrogen receptor (ER)-positive breast cancer (between $\mathrm{P}<0.05$ and $0.0001)$. The pooled analysis indicated that the most significant pathway was $G_{1} / S$ checkpoint regulation, and the cyclin and cell cycle regulation pathway for BPA-associated ER-positive cancer. Cancer cell signaling pathways were associated with healthy breast cells developing into breast cancer. The visceral obesity and the cell cycle pathways were indicated to link BPA exposure to breast cancer. The results of the present study demonstrate a significant association between breast cancer and BPA-regulated gene pathways.

\section{Introduction}

Bisphenol A (BPA) is known endocrine-disrupting chemical $(1,2)$. Previous studies have investigated exposure to BPA as a leading cause of the development of endocrine diseases, including breast cancer (1-5). The use of BPA has been identified in a variety of consumer products, including water bottles, food/beverage containers and linings, thermal receipts, medical equipment and paper cups $(6,7)$. It has been reported that the use of BPA-containing products introduces BPA into the food chains of industrialized countries (8). It has been demonstrated that $95 \%$ of BPA may be absorbed by the intestinal tract, when BPA enters the human body (9). It has been indicated that BPA binds to glucuronic acid in the liver subsequent to being metabolized through the phase II pathway $(9,10)$.

It has been demonstrated that fetal BPA exposure increases the induction of mammary gland ductal hyperplasia and carcinoma in situ (3). In a previous study, it was identified that when pregnant mice were treated with various doses $(0-250 \mu \mathrm{g} / \mathrm{kg})$ of BPA, a change in the morphology of the mammary gland was observed, possibly due to BPA, in addition to mammary tumorigenesis indicated in the offspring during adulthood $(1,11-13)$. It 
has been demonstrated that perinatal exposure to BPA changes the long-term progesterone response and increases the adult mice mammary cell number, resulting in the development of breast cancer (4). Previous studies have determined specific molecular alterations in the presence of BPA in high-risk breast tissues. They used non-malignant random periareolar fine-needle aspirates from unafflicted and contralateral breast tissue of the primary breast lesion and determined that the BPA response profile was significantly associated with breast tumors that were characterized by an advanced histological grade, large tumor size and poor patient survival rate (14-16). Another study identified that BPA increased antioxidant proteins, including B-cell lymphoma 2 apoptosis regulator and superoxidase dismutase 1 , and activated epidermal growth factor receptor pathways, inducing inflammatory breast cancer proliferation (17). Forkhead box protein A1 (FOXA1) has been identified as a hormone-response mediator in breast cancer (18). It has been identified that BPA may downregulate FOXA1, inducing mesenchymal transition and promoting the in vitro motility of estrogen receptor (ER)-negative breast cancer cells (19).

In rodents, perinatal exposure to low doses of BPA has been demonstrated to be associated with weight gain and the metabolic syndrome (MetSyn) when fed on a normal diet and more severe metabolic diseases when fed on a high-fat diet (20). Urinary BPA has been indicated to be associated with general and central obesity (21), and may be further associated with Type 2 diabetes mellitus $(22,23)$.

The aforementioned trends are generating the so-called 'diabesity phenotype' (24). A previous study indicated that, owing to the pregnant mice's exposure to BPA, the male offspring presented symptoms of diabesity (25). Another study indicated that BPA may disrupt the functions of pancreatic $\beta$-cells, adipocytes and hepatocytes, which are all targets of BPA, indicating an association with metabolic diseases, including diabesity (26). Williams (27) indicated that exposure to xenoestrogens, including BPA, may result in obesity, Type 2 diabetes, breast cancer, uterine overgrowth and prostate cancer. Obesity has also been reported to be associated with breast cancer (28). It has been identified that central obesity may be associated with breast cancer in premenopausal women (28). It has been reported that the presence of high blood pressure, insulin resistance and visceral obesity may constitute as a MetSyn, thus suggesting an association with aggressive breast cancer (29). Visceral adiposity and MetSyn have been indicated to be associated with increased tumor aggressiveness and distant metastasis of breast cancer $(30,31)$.

It has been suggested that BPA may exacerbate the risk of obesity, which may lead to an increase in breast cancer. Therefore, we hypothesized that BPA-associated pathways and candidate genes are developmentally active in breast cells, and that these molecular markers are associated with breast cancer susceptibility and aggressiveness. To test the aforementioned hypothesis, microarray datasets from an identical platform were used to determine gene sets that are associated with the development and aggressiveness of various types of breast cancer. A total of three different statistical comparison procedures were applied to test the hypothesis. Common gene sets were identified, which were significantly expressed by comparing BPA-exposed cells with breast tissue datasets and identified potential pathways that associate BPA exposure with breast cancer. In addition, the common gene sets that were associated with BPA-regulated high-grade breast cancer in different patient datasets were assessed. The probes of BPA-associated significant gene sets were further pooled to identify significant canonical pathways in various types of breast cancer.

\section{Materials and methods}

Microarray datasets. The six selected datasets, GSE50705 (32), GSE32158 (33), GDS4114 (34), GDS3853 (35), GSE5460 (36) and GSE6532 (37), used an identical gene expression platform obtained using a Human Genome U133 Plus 2.0 software array (GPL570 platform; Affymetrix; Thermo Fisher Scientific, Inc., Waltham, MA, USA) and were organized into three types. The first type consisted of two datasets for breast cancer MCF-7 and MCF-10 cell lines treated with BPA. ER-positive MCF-7 cells were exposed to $1,0.5$ and $0.25 \mu \mathrm{M}$ BPA for $48 \mathrm{~h}$ and were monitored on arrays obtained from GSE50705 (32). The normal breast epithelial MCF-10F cell line was exposed to 10 and $1 \mu \mathrm{M}$ BPA for 2 weeks and cells were monitored on arrays obtained from GSE32158 (33). The second type consisted of two breast tissue datasets, GDS4114 and GDS3853, associated with the microarray expression profiles. GDS4114 consisted of six samples of stroma-surrounding invasive type of breast tumor and six matched samples of normal stroma. Host tissue stroma interacting with tumor cells has been identified to be associated with tumor progression and metastasis (34). GDS3853 consisted of 14 samples of nine ductal carcinoma in situ and five healthy tissue samples (35). The third type consisted of two high-grade tissue datasets, GSE5460 and GSE6532. The GSE5460 microarray profile consisted of 129 patients with a tumor, including 76 ER-positive patients and 53 ER-negative patients, which were divided into 70 high-grade and 59 low-grade tumor samples (36). GSE6532 consisted of 16 high-grade and 54 low-grade ER-positive tumor samples from patients undergoing tamoxifen treatment (37).

The present study was approved by the Institutional Review Board of Kaohsiung Medical University (Kaohsiung, Taiwan). All microarray profiles were obtained from the NCBI Gene Expression Omnibus (GEO) database (www.ncbi.nlm.nih. gov/geo) from original submitter-supplied records, including series, samples and platforms, and curated datasets.

Bioinformatics and statistical methods. The normalization method of the Microarray Suite software (version 5.0; Affymetrix; Thermo Fisher Scientific, Inc.) was implemented, according to the manufacturer's protocols. The BRB-Array Tools software Beta 2 (version 4.3.0; linus. nci.nih.gov/BRB-ArrayTools.html) was utilized in the Bioconductor software suite, based on the $\mathrm{R}$ programming language $(38,39)$. The array tool program obtained the curated Biocarta pathways from the Cancer Genome Anatomy Project (cgap.nci.nih.gov/Pathways). For the gene set analysis, the least squares/Kolmogorov-Smirnov permutation test (40), Efron-Tibshirani's Gene Set Analysis maxmean test and the 1000-permutation statistics enrichment method were employed to identify significant gene sets. The cut-off level for determining significant gene sets was 0.05 . 
Table I. Significant gene set pathways from six microarray datasets.

\begin{tabular}{llcc} 
Microarray dataset & \multicolumn{1}{c}{ Exposure } & $\begin{array}{c}\text { No. of significant gene sets/total } \\
\text { number of gene sets investigated }\end{array}$ & Overlapping gene sets $^{\mathrm{a}}$ \\
\hline GSE50705 & 0.25 vs. $0 \mu \mathrm{M} \mathrm{BPA}$ & $60 / 271$ & 42 \\
GSE32158 & 0.5 vs. $0 \mu \mathrm{M} \mathrm{BPA}$ & $55 / 271$ & 72 \\
& 1 vs. $0 \mu \mathrm{M} \mathrm{BPA}$ & $66 / 271$ & - \\
GDS4114 & 1 vs. $0 \mu \mathrm{M} \mathrm{BPA}$ & $140 / 279$ & - \\
GDS3853 & 10 vs. $0 \mu \mathrm{M} \mathrm{BPA}$ & $105 / 279$ & 31 \\
GSE5460 & Breast cancer vs. healthy control & $83 / 295$ & - \\
GSE6532 & Breast cancer vs. healthy control & $77 / 295$ & - \\
\hline
\end{tabular}

${ }^{a}$ Calculated by LS/KS permutation test and Efron-Tibshirani's Gene Set Analysis maxmean test. The cut-off level for determining significant gene sets was 0.05 .

In the ER-positive MCF-7 cell line microarray, a total of 42 common gene sets were identified when exposed to 0.25 , 0.5 and $1 \mu \mathrm{M}$ BPA, with each treatment containing, out of the 271 investigated significant gene sets, 60, 55 and 66 significant gene sets, respectively (Table I). In the normal breast epithelial cell MCF-10F microarray, a total of 72 common gene sets were identified when exposed to 1 and $10 \mu \mathrm{M} \mathrm{BPA}$, with each condition containing, out of the 279 investigated significant gene sets, 140 and 105 gene sets, respectively. However, two breast tissue profiles, GDS4114 and GDS3853, contained 83 and 77 of the investigated significant gene sets, respectively (Table I). In the primary and untreated breast cancer array GSE5460, 31 overlapping gene sets, from the 50 and 53 significant gene sets, were obtained from the axillary grade (high vs. low) and ER status (positive vs. negative) assessments, respectively. GSE6532 consisted of 73 significant gene sets from ER-positive groups, comparing the high vs. low grade under tamoxifen treatment (Table I).

Two class comparisons were sequentially performed with a two-sample t-test and a gene set expression comparison analysis to identify individual candidate genes. The following fold changes were separately assessed under the supervised methods: BPA exposure vs. the control $(0 \mu \mathrm{M})$; tumor vs. normal tissue; ER-positive vs. ER-negative and high-grade (grade III) vs. low-grade (grade I) (36). The selection criteria was a fold-change cut-off value $\geq 2$ which indicated upregulation, and $\leq 0.5$ indicating downregulation between the two classes, as previously described (41). This fold change was considered significant at $\mathrm{P}<0.01$ as previously described (42). Furthermore, Entrez IDs of the genes without current gene symbols were also excluded.

Ingenuity Pathway Analysis (IPA). The candidate genes originated from the significant Biocarta pathways in the gene set analysis and the overlap of individual candidate genes from the comparisons of breast cancer datasets of BPA-treated cells. The probes of BPA-associated significant gene sets were further pooled to present a cellular pathway in breast cancer. IPA (2017 release; www.ingenuity.com) was performed to determine the significant canonical pathway. The pathway was determined and ranked on the basis of the ratio of the number of genes matched to the curated pathways in the IPA knowledge database and the P-value was determined by Fisher's exact test. The molecular network indicated that relevant associations were connected to biological interactions. The score for the aforementioned network is the negative logarithm of the P-value calculated with the right-tailed Fisher's exact test with a hypergeometric distribution.

\section{Results}

Gene sets associated with BPA-associated breast cancer from individual datasets. The gene sets, which were associated with BPA exposure were assessed in the ER-positive breast cancer cell line MCF-7, using the BRB-Array Tool and analyzed on the basis of a signaling network database of the Biocarta pathways. It was indicated that seven gene sets significantly overlapped between the MCF-7 cells exposed to BPA and the stroma of breast cancer profile GDS4114. In addition, there were 18 overlapping gene sets between the MCF-7 cells exposed to BPA and the breast cancer profile GDS3853 (Fig. 1A). Furthermore, two consistent gene sets overlapped among all three datasets: The visceral obesity pathway, involved in visceral fat deposits and MetSyn, and the cell cycle pathway, involved in cyclins and cell cycle regulation (Table II).

It was identified that 22 gene sets significantly overlapped between the normal breast epithelial cell line, MCF-10F, exposed to BPA and the stroma of breast cancer profile GDS4114, in addition to 36 overlapping gene sets between the MCF-10F cells exposed to BPA and the breast cancer profile GDS3853 (Fig. 1B). Furthermore, the following 8 consistent gene sets overlapped among all three datasets: The thrombospondin 1 (TSP-1) pathway, where TSP-1-induced apoptosis in microvascular endothelial cells; the CCCTC-binding factor pathway (first multivalent nuclear factor); the extrinsic pathway (extrinsic prothrombin activation pathway); the reversion inducing cysteine-rich protein with Kazal motifs pathway (inhibition of matrix metalloproteinases); the longevity 

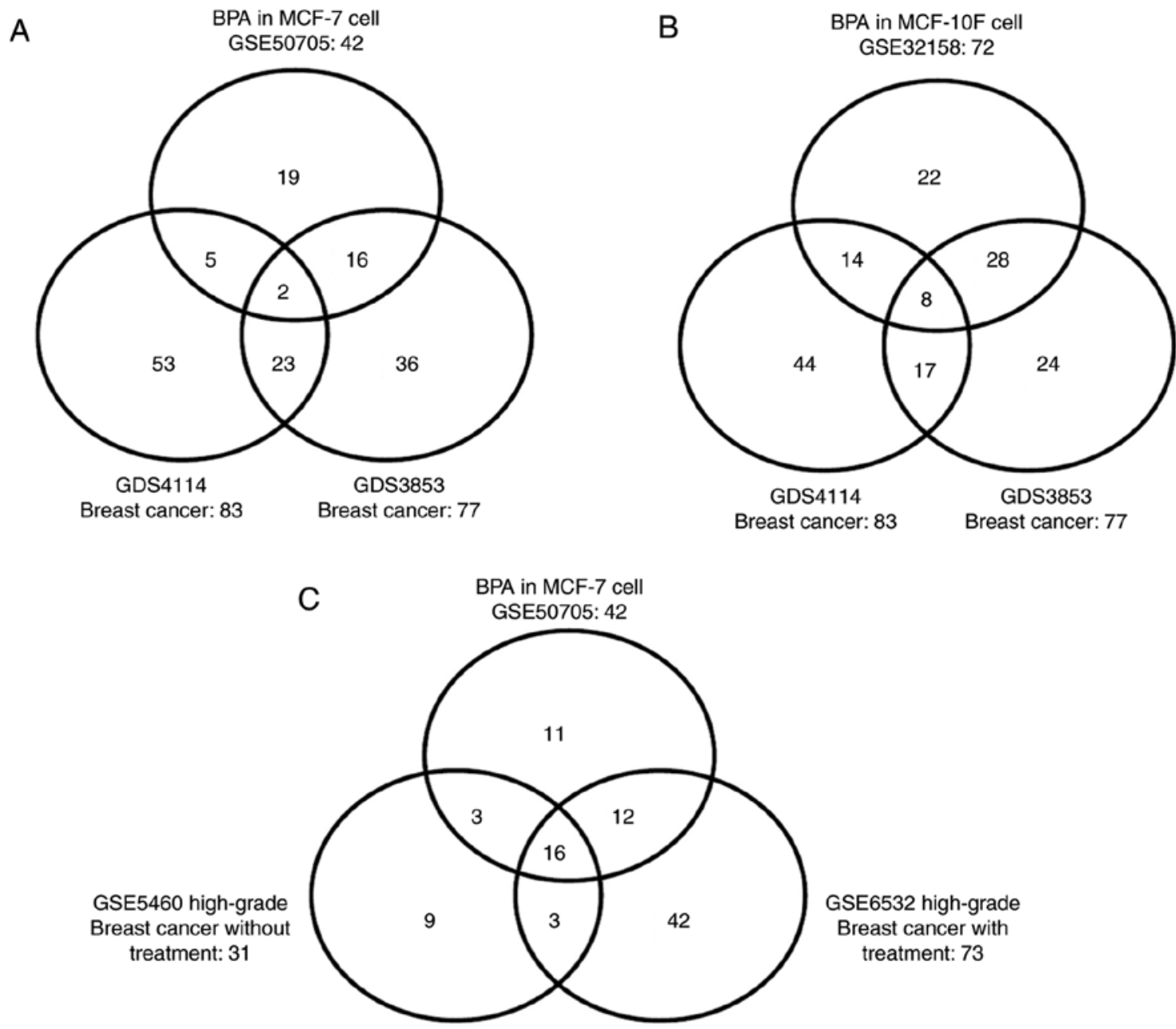

Figure 1. Gene sets associated with the development and aggressiveness of BPA-associated breast cancer. Venn diagrams illustrate (A) the overlap of gene sets using GSE50705 (MCF-7 breast cancer cell lines treated with three different doses of BPA), GDS4114 (the stroma of breast cancer), and GDS3853 (breast cancer); (B) the intersection of three different cell lines: GSE32158 (MCF-10F normal breast epithelial cell line treated with two different doses of BPA), GDS4114 and GDS3853; and (C) the results of GSE50705 intersected by aggressive breast cancer, GSE5460 and GSE6532. BPA, bisphenol A.

pathway (insulin-like growth factor 1 receptor and longevity); the acetylcholine pathway (role of nicotinic acetylcholine receptors in the regulation of apoptosis); the visceral obesity pathway (visceral fat deposits and MetSyn), and the cell cycle pathway (cyclins and cell cycle regulation) (Table II).

It was subsequently determined whether BPA-affected gene sets were associated with aggressiveness in breast cancer. A total of 16 gene sets, including the cyclins and cell cycle regulation pathway, significantly overlapped among all three datasets of MCF-7 cells exposed to BPA, and the high-grade breast cancer profiles GSE5460 and GSE6532 (Fig. 1C; Table II). In addition, the following 12 gene sets were identified in BPA-associated aggressive tumors treated with tamoxifen: Breast cancer-associated 1 (BRCA1), DNA repair associated-dependent ubiquitin-ligase activity (h_bard1Pathway); apoptotic DNA fragmentation and tissue homeostasis (h_DNAfragmentPathway); ataxia telangiectasia mutated (ATM) serine/threonine kinase signaling pathway (h_atmPathway); progesterone initiation of oocyte maturation (h_mPRPathway); stathmin and breast cancer resistance to antimicrotubular agents ( $\mathrm{h} \_$stathminPathway); Fas cell-surface death receptor signaling pathway (h_fasPathway); 553 signaling pathway (h_p53Pathway); erythrocyte differentiation pathway
(h_erythPathway); signal transduction through interleukin 1 receptor (IL1R) (h_il1rPathway); the function of small leucine-rich proteoglycan in bone (h_slrp2Pathway); nuclear factor- $\mathrm{KB}$ activation by non-typeable Haemophilus influenzae (h_nthiPathway) and the visceral fat deposits and MetSyn pathway (h_vobesityPathway). Therefore, the visceral fat deposits and MetSyn pathway (h_vobesityPathway), and the cyclins and cell cycle regulation (cell cycle pathway) were involved in BPA-associated breast cancer development and aggressiveness (Table II).

Pooled analysis of the gene sets in the development and aggressiveness of BPA-associated breast cancer. The probes of BPA-associated significant gene sets were further pooled to present a cellular pathway using IPA. The most significant cellular pathway of ER-positive breast cancer was the cell cycle $\mathrm{G}_{1} / \mathrm{S}$ checkpoint regulation (with GDS4114, $\mathrm{P}=3.63 \times 10^{-55}$ and with GDS3853, $\mathrm{P}=2.40 \times 10^{-28}$ ), whereas other statistically significant cellular pathways were associated with cyclin and cell cycle regulation (with GDS4114, $\mathrm{P}=7.76 \times 10^{-52}$ and with GDS3853, $P=1.74 \times 10^{-35}$ ), cell cycle $\mathrm{G}_{2} / \mathrm{M}$ damage check point regulation (with GDS3853, $\mathrm{P}=2.14 \times 10^{-37}$ ), aryl hydrocarbon receptor signaling (with GDS4114, $\mathrm{P}=3.63 \times 10^{-40}$ 
Table II. Significant pathways that link BPA exposure to breast cancer.

A, MCF-7 (GSE50705), GDS4114 and GDS3853

\begin{tabular}{lllrr}
\hline No. & Biocarta pathway & Pathway description & No. of probes & P-value $^{\mathrm{a}}$ \\
\hline 1 & h_cellcyclePathway & Cyclins and cell cycle regulation & 29 & $1.00 \times 10^{-5}-5.00 \times 10^{-3}$ \\
2 & h_vobesityPathway & Visceral fat deposits and the metabolic syndrome & 7 & $1.00 \times 10^{-5}-1.29 \times 10^{-2}$ \\
\hline
\end{tabular}

B, MCF-10F (GSE32158), GDS4114 and GDS3853

\begin{tabular}{|c|c|c|c|c|}
\hline No. & Biocarta pathway & Pathway description & No. of probes & P-value \\
\hline 1 & h_tsp1Pathway & $\begin{array}{l}\text { TSP-1 induced apoptosis in microvascular } \\
\text { endothelial cells }\end{array}$ & 16 & $1.00 \times 10^{-5}-6.51 \times 10^{-3}$ \\
\hline 2 & h_ctcfPathway & CTCF: First multivalent nuclear factor & 47 & $1.00 \times 10^{-5}-2.44 \times 10^{-2}$ \\
\hline 3 & h_extrinsicPathway & Extrinsic prothrombin activation pathway & 9 & $2.00 \times 10^{-5}-2.42 \times 10^{-3}$ \\
\hline 4 & h_reckPathway & Inhibition of matrix metalloproteinases & 14 & $<1.00 \times 10^{-3}-2.33 \times 10^{-2}$ \\
\hline 5 & h_vobesityPathway & Visceral fat deposits and the metabolic syndrome & 11 & $1.00 \times 10^{-5}-1.29 \times 10^{-2}$ \\
\hline 6 & h_longevityPathway & IGF-1 receptor and longevity & 19 & $1.00 \times 10^{-5}-2.89 \times 10^{-3}$ \\
\hline 7 & h_achPathway & $\begin{array}{l}\text { Role of nicotinic acetylcholine receptors in the } \\
\text { regulation of apoptosis }\end{array}$ & 13 & $<1.00 \times 10^{-3}-4.24 \times 10^{-2}$ \\
\hline 8 & h_cellcyclePathway & Cyclins and cell cycle regulation & 33 & $1.00 \times 10^{-5}-5.00 \times 10^{-3}$ \\
\hline
\end{tabular}

C, MCF7 (GSE50705), GDS6532 and GDS5460 with high grade

\begin{tabular}{|c|c|c|c|c|}
\hline No. & Biocarta pathway & Pathway description & No. of probes & P-value ${ }^{a}$ \\
\hline 1 & h_cellcyclePathway & Cyclins and cell cycle regulation & 29 & $1.00 \times 10^{-5}-1.10 \times 10^{-4}$ \\
\hline 2 & h_EfpPathway & EFP controls the cell cycle and breast tumor growth & 28 & $1.00 \times 10^{-5}-4.75 \times 10^{-3}$ \\
\hline 3 & h_g1Pathway & Cell cycle: $\mathrm{G}_{1} / \mathrm{S}$ checkpoint & 49 & $1.00 \times 10^{-5}-2.29 \times 10^{-3}$ \\
\hline 4 & h_g2Pathway & Cell cycle: $\mathrm{G}_{2} / \mathrm{M}$ checkpoint & 40 & $1.00 \times 10^{-5}-1.80 \times 10^{-2}$ \\
\hline 5 & h_mcmPathway & CDK regulation of DNA replication & 31 & $1.00 \times 10^{-5}-1.00 \times 10^{-5}$ \\
\hline 6 & h_rbPathway & $\begin{array}{l}\mathrm{Rb} \text { tumor suppressor/checkpoint signaling in } \\
\text { response to DNA damage }\end{array}$ & 24 & $1.00 \times 10^{-5}-1.80 \times 10^{-2}$ \\
\hline 7 & h_cdc25Pathway & $\begin{array}{l}\text { CDC25 and CHK1 regulatory pathway in response } \\
\text { to DNA damage }\end{array}$ & 18 & $1.00 \times 10^{-5}-1.00 \times 10^{-3}$ \\
\hline 8 & h_ptc1Pathway & Sonic Hedgehog receptor PTC1 regulates cell cycle & 16 & $1.00 \times 10^{-5}-7.76 \times 10^{-3}$ \\
\hline 9 & h_ranMSpathway & Role of Ran in mitotic spindle regulation & 20 & $1.00 \times 10^{-5}-1.52 \times 10^{-3}$ \\
\hline 10 & h_p27Pathway & $\begin{array}{l}\text { Regulation of p } 27 \text { phosphorylation during cell } \\
\text { cycle progression }\end{array}$ & 22 & $5.00 \times 10^{-5}-5.73 \times 10^{-3}$ \\
\hline 11 & h_atrbrcaPathway & $\begin{array}{l}\text { Role of BRCA1, BRCA2 and ATR in cancer } \\
\text { susceptibility }\end{array}$ & 45 & $5.30 \times 10^{-4}-1.75 \times 10^{-2}$ \\
\hline 12 & h_srcRPTPPathway & Activation of Src by protein tyrosine phosphatase $\alpha$ & 17 & $1.00 \times 10^{-5}-6.45 \times 10^{-3}$ \\
\hline 13 & h_skp2e2fPathway & E2F1 destruction pathway & 18 & $4.60 \times 10^{-4}-8.06 \times 10^{-3}$ \\
\hline 14 & h_akap95Pathway & $\begin{array}{l}\text { Role of AKAP95 in mitosis and chromosome } \\
\text { dynamics }\end{array}$ & 18 & $<1.00 \times 10^{-3}-3.75 \times 10^{-2}$ \\
\hline 15 & h_plk3Pathway & Regulation of cell cycle progression by PLK3 & 15 & $2.00 \times 10^{-5}-3.90 \times 10^{-2}$ \\
\hline 16 & h_fbw7Pathway & Cyclin E destruction pathway & 19 & $7.97 \times 10^{-3}-4.81 \times 10^{-2}$ \\
\hline
\end{tabular}

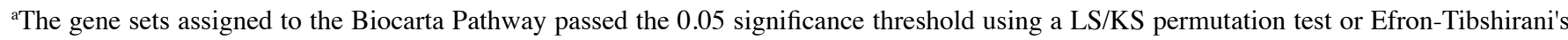
Gene Set Analysis maxmean test (under 1000 permutations). BPA, bisphenol A; TSP-1, thrombospondin 1; CTCF, CCCTC-binding factor; IGF-1, insulin-like growth factor 1; CDK, cyclin-dependent kinase; RB, retinoblastoma; CDC25, cell division cycle 25C; CHK1, checkpoint kinase 1; BRCA, breast cancer-associated; ATR, ataxia telangiectasia and Rad3-related; E2F1, E2F transcription factor 1; AKAP95, A-kinase-anchoring protein 8; PLK3, Polo-like kinase 3; EFP, estrogen-responsive protein; PTC1, patched 1. 

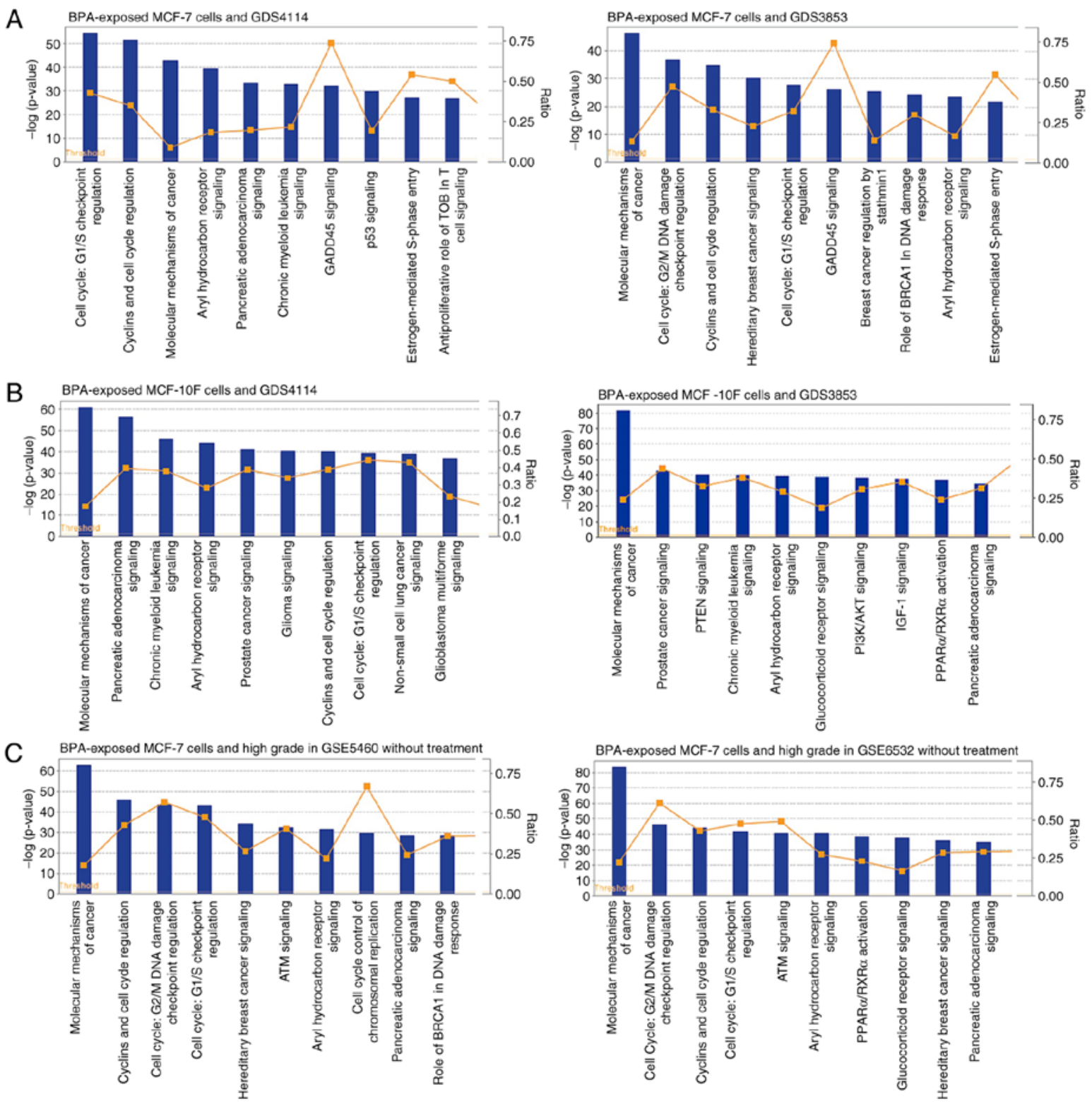

Figure 2. Cellular canonical pathways associated with the development and high grade of breast cancer. (A) The results of BPA-exposed MCF-7 cells (GSE50705), GDS4114 and GDS3853. (B) The results of BPA-exposed MCF-10F cells (GSE32158), GDS4114 and GDS3853. (C) The results of BPA-exposed MCF-7 cells (GSE50705), GSE5460 (high grade without treatment) and GSE6532 (high grade with treatment). The left $y$-axis indicates the negative logarithm of the P-value calculated using a right-tailed Fisher's exact test with the threshold set at $\mathrm{P}<0.05$. The right $y$-axis indicates the ratios of the number of genes in the pathway that meet the cut-off criteria to the total number of genes in that pathway. BPA, bisphenol A; GADD45, growth arrest and DNA damage-inducible 45; TOB, transducer of ERBB2; BRCA, breast cancer-associated; IGF-1, insulin-like growth factor 1; PTEN, phosphatase and tensin homolog; AKT, protein kinase B; PPAR $\alpha$, peroxisome-proliferator-activated receptor $\alpha$; ATM, ataxia telangiectasia mutated.

and with GDS3853, $\left.\mathrm{P}=4.68 \times 10^{-24}\right)$ and hereditary breast cancer signaling (with GDS3853, $\mathrm{P}=1.00 \times 10^{-30}$ ) (Fig. 2A). The most significant cellular pathway from gene sets of normal breast cells and breast cancer was the 'molecular mechanisms of cancer' (with GDS4114, $\mathrm{P}=2.34 \times 10^{-61}$ and with GDS3853, $\mathrm{P}=2.14 \times 10^{-82}$ ) (Fig. 2B) from the cellular canonical pathways analysis. It was subsequently determined whether BPA-regulated cellular pathways were associated with high-grade breast cancer. The most significant cellular pathway for the ER-positive and high-grade breast cancer was the 'molecular mechanisms of cancer' (with GSE5460, $\mathrm{P}=3.16 \times 10^{-63}$ and with GSE6532, $\mathrm{P}=4.68 \times 10^{-84}$ ) (Fig. 2C). Numerous cell cycle pathways, including cyclin and cell cycle regulation, $\mathrm{G}_{2} / \mathrm{M}$ damage and $\mathrm{G}_{1} / \mathrm{S}$ checkpoint regulation, for the ER-positive and high-grade breast cancer were also observed (Fig. 2C).

Biological interaction analysis of the gene sets. Biological interaction maps of genes that are commonly expressed in human breast cells and breast tissue following BPA exposure were generated using IPA. Simplified versions of two network maps for the visceral obesity pathway and cell cycle pathway are presented in Fig. 3. A multiple interaction network of the visceral obesity pathway was involved in visceral fat deposits and MetSyn, revealing adiponectin, retinoid $\mathrm{X}$ receptor $\alpha$ and nuclear receptor subfamily 3 group C member 1 (Fig. 3A). The network interaction map for the cell cycle pathway identified a number of genes with multiple interactions that were 

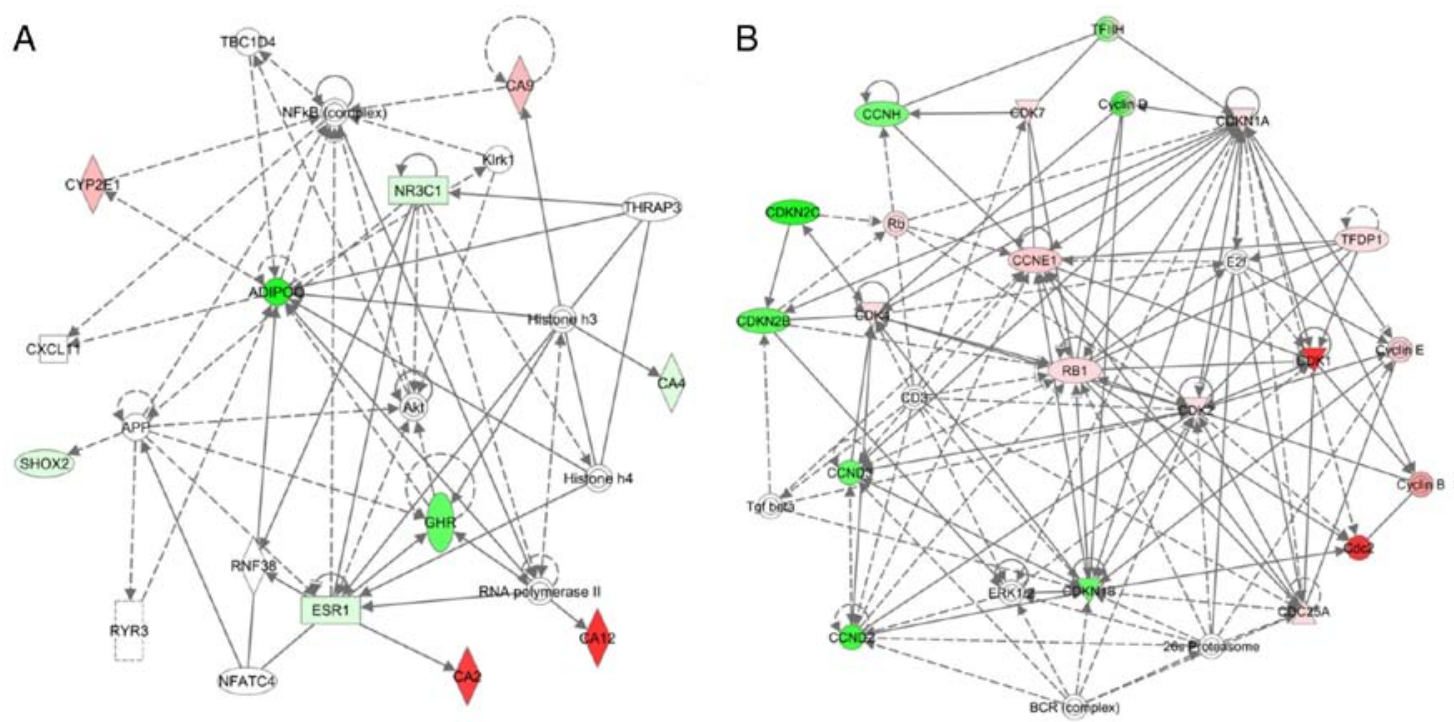

Figure 3. Interaction network analysis of the (A) visceral obesity pathway and (B) the cell cycle pathway, using the path designer of IPA. A simplified version of IPA pathway maps generated for genes that were commonly expressed in microarrays of human breast cells and breast tissue treated with BPA. Red and green nodes represent upregulated or downregulated genes. White nodes represent the genes in a pathway not available in the microarray for testing. Symbols represent the following: Concentric circle, complex/group; square, cytokine; rhombus, enzyme; dashed rectangle, ion channel; inverted triangle, kinase; rectangle, ligand-dependent nuclear receptor; circle, other; triangle, phosphatase; horizontal oval, transcription regulator; vertical oval, transmembrane receptor. BPA, bisphenol A; IPA, Ingenuity Pathway Analysis.

associated with cyclins and cell cycle regulation, including cyclin-dependent kinases 1, 2 and 4, cyclin-dependent kinase inhibitors 1A, 2B and 2C, cyclin B1, E2F transcription factor 1 and retinoblastoma 1 (Fig. 3B).

\section{Discussion}

The aim of the present study was to identify the potential pathways of the progression from normal breast epithelial cells to the development and aggressiveness of breast cancer following exposure to BPA. The two BPA-treated datasets and the two breast tissue datasets all displayed an overlap of two consistent gene sets: The visceral obesity pathway, involved in visceral fat deposits and MetSyn, and the cell cycle pathway, involved in cyclins and cell cycle regulation. Visceral adipose tissue has been recognized as an important metabolic tissue, secreting factors that may alter metabolism and immunity (43). Visceral obesity, insulin resistance and pro-inflammatory state were defined as MetSyn. It has been identified that MetSyn was associated with the pathogenesis of breast cancer and colorectal cancer (43). It has been demonstrated that cyclin D1 serves as a cell cycle regulatory switch and is important in the regulation of proliferation during mammary oncogenesis (44). Cyclin-dependent kinase 4 (CDK4) and CDK6 are proteins in the cell cycle regulatory pathway (45). When there is an imbalance in the cyclin D and cyclin-dependent kinase (CDK) pathway in cancer cells, the cells may progress towards a more proliferative phenotype. The present study demonstrated that oral CDK inhibitors may improve the outcomes in patients with ER-positive breast cancer (45). It has been reported that the R-point is important in cell cycle control and it switches on the $\mathrm{G}_{1}$-to-S transition process (46). It has been demonstrated that if the cells pass the R-point, they can subsequently evade apoptosis, proliferation and metastasis (46). The results of the present study suggest that BPA-affected gene sets cause breast carcinogenesis, through the visceral fat deposits and MetSyn pathway, and the cell cycle pathway. In addition, the aforementioned processes lead to high-grade aggressiveness in breast cancer. The visceral fat deposits and MetSyn pathway, and the cell cycle pathway may be involved when BPA affects high-grade tumor aggressiveness.

Previous studies have indicated that BPA causes obesity and metabolic disruption in animals and humans (21-23). The National Health and Nutrition Examination Surveys reported that urinary BPA concentrations are positively associated with general and central obesity (21). It has been suggested that exposure to BPA may lead to insulin resistance and the development of Type 2 diabetes mellitus, which may occur through the overactivation of pancreatic $\beta$-cells $(22,23)$. A previous study conducted in a Chinese population identified an association among urinary BPA concentrations and obesity, insulin resistance, and disturbances in body weight regulation (47). The aforementioned results are consistent with the results of the present study in which exposure to BPA initiated the visceral obesity pathway, involved in visceral fat deposits and MetSyn. A murine study identified that BPA injections into the fat pads enhanced epithelial tumor proliferation rather than subcutaneous proliferation (48). Furthermore, a number of cohort studies have indicated that abdominal obesity and MetSyn increased the risk of breast cancer (49-51). It has been identified that numerous inflammatory cells in the visceral adipose tissue, including macrophages and $\mathrm{T}$ cells, generate a pro-tumorigenic environment (43).

A proteomic analysis of mammary fat identified numerous signaling molecules involved in a variety of biological processes, including signal transduction and cell communication, energy and protein metabolism, proliferation and apoptosis, all of which served an important function in the breast tumor microenvironment (52). The underlying molecular mechanisms of breast carcinogenesis by visceral adiposity and MetSyn have 
been indicated to include microenvironmental alterations in cell signaling pathways and adipokine secretion (43). A gene set analysis of BPA, combined with $17 \beta$-estradiol and progesterone exposure in eight non-malignant breast epithelial cell culture samples, elucidated six biological pathways implicated in tumorigenesis. Exposure to BPA led to the overexpression of genes facilitating cell cycle progression and created a high-risk microenvironment for breast tumor development and aggressiveness (14). The results of the present study support those of the aforementioned previous studies. In the present study, it was identified that the cell cycle pathway involved in cyclins and cell cycle regulation is associated with BPA-regulated breast carcinogenesis.

It has been reported that breast cancer consists of multiple different subtypes and biological processes, and therefore distinct gene set pathways are associated with prognosis and chemotherapy sensitivity in these disease subsets (53). In the individual dataset analysis for the aforementioned biological pathways, it was indicated that two pathways overlapped among the predictive gene sets in the BPA-treated cells and breast cancer datasets. This result indicated that different cellular pathways following BPA exposure were associated with the progression of normal breast cells to breast cancer cells. From the pooled analysis, the gene sets involved in the pathways of $\mathrm{G}_{2} / \mathrm{M}$ DNA damage, growth arrest and DNA damage-inducible 45, p53 and T-cell signalling, and estrogen-mediated $\mathrm{S}$ phase entry were associated with BPA-regulated carcinogenesis of ER-positive breast cancer. A previous review of numerous multiple oncogenic signaling pathways following BPA exposure in ER-positive breast cancer cells identified vascular endothelial growth factor signaling, the DNA repair pathway, extracellular-signal-regulated kinase $1 / 2$ activation and signal transducer and activator of transcription 3 signaling (54). Furthermore, DNA damage, involving cell cycle $\mathrm{G}_{2} / \mathrm{M}$ and BRCA1, ATM and hereditary breast cancer signaling gene sets were associated with BPA-regulated high-grade aggressiveness. The result is consistent with a functional study that indicated that gene expression alteration, due to BPA, was significantly associated with an advanced histological grade and large tumor size in breast tumors (14).

In addition to the breast cancer cell line MCF-7, the normal breast epithelial cell line MCF-10F was also selected to mimic the cells of healthy subjects exposed to BPA. In the present study, the gene set analysis of MCF-10F exposed to BPA compared with breast cancer datasets elucidated two important biological pathways, the visceral fat deposits and MetSyn pathway, and cyclins and cell cycle regulation, which are implicated in tumorigenesis associated to BPA. The stimulation of immortalized breast cells suggests a possible function for these pathways in modulating the carcinogenesis and aggressiveness of breast cancer. The microarray association results indicate that exposure to BPA initiates processes that are a part of the breast cancer developmental cycle. The combination of the aforementioned results with clinical and epidemiologic evidence associates BPA exposure with subsequent breast cancer.

A number of limitations are noted in the present study. First, the tumor datasets were from different patient cohorts with different inclusion and exclusion criteria, along with the breast cell line datasets being treated with different doses of BPA. However, the six datasets selected used an identical gene expression platform, GPL570, to decrease any bias resulting from quantifying different initial gene sets and inconsistent associative intensity values for a candidate gene. Secondly, different methodologies may have been applied in patient archiving and specimen preparation, and different pre-analytical variations may have occurred. This variability may decrease the reproducibility of the results of the present study. Thirdly, owing to the limitation of the selected platform, the sample size of the datasets and study subjects for patients with breast cancer were limited in each of the array datasets. Patients included in one high-grade dataset were treated with tamoxifen regimens, whereas patients in the other high-grade dataset received no treatment. Therefore, the probability of coincidental discoveries cannot be excluded due to the limited sample size and low statistical power.

Furthermore, the selection criteria for the searched microarray datasets from the NCBI's GEO database were BPA, Homo sapiens and gene expression array until July 6, 2017. It was indicated that five databases were breast-associated cell lines, as well as BPA-treated, two of which, GSE50705 and GSE32158, were included in the present study. The other three datasets were MCF-7 cells treated with $0.4 \mu \mathrm{M}$ BPA for $48 \mathrm{~h}$, MCF-10F cells treated with 10 and $1 \mu \mathrm{M}$ BPA, and MCF-10F cells treated with 10 and $1 \mu \mathrm{M}$ for 2 weeks. The datasets selected had a lower BPA concentration compared with the other three datasets, including GSE50705, MCF-7-treated with $0.25,0.5$ and $1 \mu \mathrm{M}$ BPA for $48 \mathrm{~h}$. However, doses of BPA were not biologically relevant in humans. Further functional studies are required to validate the predictive and prognostic value of the proposed pathways and markers of breast cancer. The results of the present study suggest that normal cell exposure to BPA may lead to breast cancer development and aggressiveness, through the regulation of key BPA pathways, particularly for the visceral obesity pathway and the cell cycle pathway.

\section{Acknowledgements}

Not applicable.

\section{Funding}

The present study was supported by the National Science Council of Taiwan (grant no. NSC 102-2632-B-037-001-MY3) and partially from the Kaohsiung Medical University 'Aim for the Top Universities Grant' (grant nos. KMU-TP103A16, KMU-TP104A01 and KMU-TP105A05).

\section{Availability of data and materials}

The datasets used and/or analyzed during the current study are available from the corresponding author on reasonable request.

\section{Authors' contributions}

TNW conceived and designed the study and drafted the manuscript. PJY, YTT and YST edited the manuscript and analyzed the datasets. PLK, CCC, SSL, THH, MFH and EMT 
were involved in the conception of the study. All authors read and approved the manuscript and agree to be accountable for all aspects of the research in ensuring that the accuracy or integrity of any part of the work are appropriately investigated and resolved.

\section{Ethics approval and consent to participate}

Not applicable.

\section{Patient consent for publication}

Not applicable.

\section{Competing interests}

The authors declare that they have no competing interests.

\section{References}

1. Weber Lozada K and Keri RA: Bisphenol A increases mammary cancer risk in two distinct mouse models of breast cancer. Biol Reprod 85: 490-497, 2011.

2. Katchy A, Pinto C, Jonsson P, Nguyen-Vu T, Pandelova M Riu A, Schramm KW, Samarov D, Gustafsson JÅ, Bondesson M and Williams C: Coexposure to phytoestrogens and bisphenol a mimics estrogenic effects in an additive manner. Toxicol Sci 138 $21-35,2014$

3. Murray TJ, Maffini MV, Ucci AA, Sonnenschein C and Soto AM Induction of mammary gland ductal hyperplasias and carcinoma in situ following fetal bisphenol A exposure. Reprod Toxicol 23 383-390, 2007.

4. Ayyanan A, Laribi O, Schuepbach-Mallepell S, Schrick C, Gutierrez M, Tanos T,Lefebvre G, Rougemont J, Yalcin-Ozuysal O and Brisken C: Perinatal exposure to bisphenol a increases adult mammary gland progesterone response and cell number. Mol Endocrinol 25: 1915-1923, 2011.

5. Jenkins S, Wang J, Eltoum I, Desmond R and Lamartiniere CA: Chronic oral exposure to bisphenol A results in a nonmonotonic dose response in mammary carcinogenesis and metastasis in MMTV-erbB2 mice. Environ Health Perspect 119: 1604-1609, 2011

6. Cooper JE, Kendig EL and Belcher SM: Assessment of bisphenol A released from reusable plastic, aluminium and stainless steel water bottles. Chemosphere 85: 943-947, 2011.

7. Geens T, Goeyens L, Kannan K, Neels H and Covaci A: Levels of bisphenol-A in thermal paper receipts from Belgium and estimation of human exposure. Sci Total Environ 435-436: 30-33, 2012

8. Sutton P, Wallinga D, Perron J, Gottlieb M, Sayre L and Woodruff T: Reproductive health and the industrialized food system: A point of intervention for health policy. Health Aff (Millwood) 30: 888-897, 2011.

9. Dekant W and Völkel W: Human exposure to bisphenol A by biomonitoring: Methods, results and assessment of environmental exposures. Toxicol Appl Pharmacol 228: 114-134, 2008.

10. Völkel W, Colnot T, Csanády GA, Filser JG and Dekant W: Metabolism and kinetics of bisphenol a in humans at low doses following oral administration. Chem Res Toxicol 15: 1281-1287, 2002

11. Acevedo N, Davis B, Schaeberle CM, Sonnenschein C and Soto AM: Perinatally administered bisphenol a as a potential mammary gland carcinogen in rats. Environ Health Perspect 121: 1040-1046, 2013.

12. Vandenberg LN, Schaeberle CM, Rubin BS, Sonnenschein C and Soto AM: The male mammary gland: A target for the xenoestrogen bisphenol A. Reprod Toxicol 37: 15-23, 2013.

13. Dhimolea E, Wadia PR, Murray TJ, Settles ML, Treitman JD, Sonnenschein C, Shioda T and Soto AM: Prenatal exposure to BPA alters the epigenome of the rat mammary gland and increases the propensity to neoplastic development. PLoS One 9: e99800, 2014.

14. Dairkee SH, Seok J, Champion S, Sayeed A, Mindrinos M, Xiao W, Davis RW and Goodson WH: Bisphenol A induces a profile of tumor aggressiveness in high-risk cells from breast cancer patients. Cancer Res 68: 2076-2080, 2008.
15. Dairkee SH,Luciani-Torres MG, Moore DH and Goodson WH III: Bisphenol-A-induced inactivation of the p53 axis underlying deregulation of proliferation kinetics, and cell death in non-malignant human breast epithelial cells. Carcinogenesis 34: 703-712, 2013.

16. Goodson WH III, Luciani MG, Sayeed SA, Jaffee IM, Moore DH II and Dairkee SH: Activation of the mTOR pathway by low levels of xenoestrogens in breast epithelial cells from high-risk women. Carcinogenesis 32: 1724-1733, 2011.

17. Sauer SJ, Tarpley M, Shah I, Save AV, Lyerly HK, Patierno SR, Williams KP and Devi GR: Bisphenol A activates EGFR and ERK promoting proliferation, tumor spheroid formation and resistance to EGFR pathway inhibition in estrogen receptor-negative inflammatory breast cancer cells. Carcinogenesis 38: 252-260, 2017.

18. Robinson JL and Carroll JS: Fox A1 is a key mediator of hormonal response in breast and prostate cancer. Front Endocrinol (Lausanne) 3: 68, 2012.

19. Zhang XL, Wang HS, Liu N and Ge LC: Bisphenol A stimulates the epithelial mesenchymal transition of estrogen negative breast cancer cells via FOXA1 signals. Arch Biochem Biophys 585: 10-16, 2015.

20. Wei J, Lin Y, Li Y, Ying C, Chen J, Song L, Zhou Z, Lv Z, Xia W, Chen $X$ and $X u$ S: Perinatal exposure to bisphenol A at reference dose predisposes offspring to metabolic syndrome in adult rats on a high-fat diet. Endocrinology 152: 3049-3061, 2011.

21. Carwile JL and Michels KB: Urinary bisphenol A and obesity: NHANES 2003-2006. Environ Res 111: 825-830, 2011.

22. Silver MK, O'Neill MS, Sowers MR and Park SK: Urinary bisphenol A and type-2 diabetes in U.S. adults: Data from NHANES 2003-2008. PLoS One 6: e26868, 2011.

23. Shankar A and Teppala S: Relationship between urinary bisphenol A levels and diabetes mellitus. J Clin Endocrinol Metab 96: 3822-3826, 2011.

24. Barouki R, Gluckman PD, Grandjean P, Hanson M and Heindel JJ: Developmental origins of non-communicable disease: Implications for research and public health. Environ Health 11: 42, 2012.

25. García-Arevalo M, Alonso-Magdalena P, Rebelo Dos Santos J, Quesada I, Carneiro EM and Nadal A: Exposure to bisphenol-A during pregnancy partially mimics the effects of a high-fat diet altering glucose homeostasis and gene expression in adult male mice. PLoS One 9: e100214, 2014.

26. Porreca I, Ulloa-Severino L, Almeida P, Cuomo D, Nardone A, Falco G, Mallardo M and Ambrosino C: Molecular targets of developmental exposure to bisphenol A in diabesity: A focus on endoderm-derived organs. Obes Rev 18: 99-108, 2017.

27. Williams GP: The role of oestrogen in the pathogenesis of obesity, type 2 diabetes, breast cancer and prostate disease. Eur J Cancer Prev 19: 256-271, 2010

28. Harvie M, Hooper L and Howell AH: Central obesity and breast cancer risk: A systematic review. Obes Rev 4: 157-173, 2003.

29. Healy LA, Ryan AM, Carroll P, Ennis D, Crowley V, Boyle T, Kennedy MJ, Connolly E and Reynolds JV: Metabolic syndrome, central obesity and insulin resistance are associated with adverse pathological features in postmenopausal breast cancer. Clin Oncol (R Coll Radiol) 22: 281-288, 2010.

30. Capasso I, Esposito E, de Laurentiis M, Maurea N, Cavalcanti E, Botti G, Petrillo A, Montella M, D'Aiuto M, Coppola C, et al: Metabolic syndrome-breast cancer link varies by intrinsic molecular subtype. Diabetol Metab Syndr 6: 105, 2014.

31. Berrino F, Villarini A, Traina A, Bonanni B, Panico S, Mano MP, Mercandino A, Galasso R, Barbero M, Simeoni M, et al: Metabolic syndrome and breast cancer prognosis. Breast Cancer Res Treat 147: 159-165, 2014

32. Shioda T, Rosenthal NF, Coser KR, Suto M, Phatak M, Medvedovic M, Carey VJ and Isselbacher KJ: Expressomal approach for comprehensive analysis and visualization of ligand sensitivities of xenoestrogen responsive genes. Proc Natl Acad Sci USA 110: 16508-16513, 2013.

33. NCBI:Bisphenol A Regulates the Expression ofDNARepairGenes in Human Breast Epithelial Cells (expression data). https://www. ncbi.nlm.nih.gov/geo/query/acc.cgi?acc=GSE32158. Accessed Septemper 16, 2011.

34. Planche A, Bacac M, Provero P, Fusco C, Delorenzi M, Stehle JC and Stamenkovic I: Identification of prognostic molecular features in the reactive stroma of human breast and prostate cancer. PLoS One 6: e18640, 2011.

35. Kretschmer C, Sterner-Kock A, Siedentopf F, Schoenegg W, Schlag PM and Kemmner W: Identification of early molecular markers for breast cancer. Mol Cancer 10: 15, 2011. 
36. Lu X, Lu X, Wang ZC, Iglehart JD, Zhang X and Richardson AL: Predicting features of breast cancer with gene expression patterns. Breast Cancer Res Treat 108: 191-201, 2008

37. Loi S, Haibe-Kains B, Desmedt C, Lallemand F, Tutt AM, Gillet C, Ellis P, Harris A, Bergh J, Foekens JA, et al: Definition of clinically distinct molecular subtypes in estrogen receptor-positive breast carcinomas through genomic grade. J Clin Oncol 25: 1239-1246, 2007.

38. Xu X, Zhao Y and Simon R: Gene set expression comparison kit for BRB-ArrayTools. Bioinformatics 24: 137-139, 2008

39. Simon R, Lam A, Li MC, Ngan M, Menenzes S and Zhao Y: Analysis of gene expression data using BRB-ArrayTools. Cancer Inform 3: 11-17, 2007.

40. Takashima S, Usui S, Kurokawa K, Kitano T, Kato T, Murai H, Furusho H, Oda H, Maruyama M, Nagata Y, et al: Altered gene expression in $\mathrm{T}$-cell receptor signalling in peripheral blood leucocytes in acute coronary syndrome predicts secondary coronary events. Open Heart 3: e000400, 2016.

41. Korkor MT, Meng FB, Xing SY, Zhang MC, Guo JR, Zhu XX and Yang P: Microarray analysis of differential gene expression profile in peripheral blood cells of patients with human essential hypertension. Int J Med Sci 8: 168-179, 2011.

42. Li Y, Li J, Fang C, Shi L, Tan J, Xiong Y, Bin Fan and Li C: Genome-wide differential expression of genes and small RNAs in testis of two different porcine breeds and at two different ages. Sci Rep 6: 26852, 2016.

43. Doyle SL, Donohoe CL, Lysaght J and Reynolds JV: Visceral obesity, metabolic syndrome, insulin resistance and cancer. Proc Nutr Soc 71: 181-189, 2012.

44. Caldon CE, Daly RJ, Sutherland RL and Musgrove EA: Cell cycle control in breast cancer cells. J Cell Biochem 97: 261-274, 2006.

45. Murphy CG and Dickler MN: The role of CDK4/6 inhibition in breast cancer. Oncologist 20: 483-490, 2015.

46. Tenga MJ and Lazar IM: Proteomic snapshot of breast cancer cell cycle: G1/S transition point. Proteomics 13: 48-60, 2013.

47. Wang T, Li M, Chen B, Xu M, Xu Y, Huang Y, Lu J, Chen Y, Wang W, Li X, et al: Urinary bisphenol A (BPA) concentration associates with obesity and insulin resistance. J Clin Endocrinol Metab 97: E223-E227, 2012.
48. Elliott BE, Tam SP, Dexter D and Chen ZQ: Capacity of adipose tissue to promote growth and metastasis of a murine mammary carcinoma: Effect of estrogen and progesterone. Int J Cancer 51: 416-424, 1992.

49. Calle EE, Rodriguez C, Walker-Thurmond K and Thun MJ: Overweight, obesity, and mortality from cancer in a prospectively studied cohort of U.S. Adults. N Engl J Med 348: 1625-1638, 2003.

50. Reeves GK, Pirie K, Beral V, Green J, Spencer E and Bull D; Million Women Study Collaboration: Cancer incidence and mortality in relation to body mass index in the Million women study: Cohort study. BMJ 335: 1134, 2007.

51. John EM, Sangaramoorthy M, Hines LM, Stern MC, Baumgartner KB, Giuliano AR, Wolff RK and Slattery ML: Overall and abdominal adiposity and premenopausal breast cancer risk among hispanic women: The breast cancer health disparities study. Cancer Epidemiol Biomarkers Prev 24: 138-147, 2015.

52. Celis JE, Moreira JM, Cabezón T, Gromov P, Friis E, Rank F and Gromova I: Identification of extracellular and intracellular signaling components of the mammary adipose tissue and its interstitial fluid in high risk breast cancer patients: Toward dissecting the molecular circuitry of epithelial-adipocyte stromal cell interactions. Mol Cell Proteomics 4: 492-522, 2005.

53. Iwamoto T, Bianchini G, Booser D, Qi Y, Coutant C, Shiang CY, Santarpia L, Matsuoka J, Hortobagyi GN, Symmans WF, et al: Gene pathways associated with prognosis and chemotherapy sensitivity in molecular subtypes of breast cancer. J Natl Cancer Inst 103: 264-272, 2011.

54. Gao H, Yang BJ, Li N, Feng LM, Shi XY, Zhao WH and Liu SJ: Bisphenol A and hormone-associated cancers: Current progress and perspectives. Medicine (Baltimore) 94: e211, 2015.

This work is licensed under a Creative Commons Attribution-NonCommercial-NoDerivatives 4.0 International (CC BY-NC-ND 4.0) License. 